\title{
A Genetic Risk Score Is Associated with Weight Loss Following Roux-en Y Gastric Bypass Surgery
}

\author{
Marcus Bandstein ${ }^{1}$ (D) - Sarah Voisin ${ }^{1} \cdot$ Emil K. Nilsson ${ }^{1} \cdot$ Bernd Schultes $^{2}$ • \\ Barbara Ernst $^{2}$ • Martin Thurnheer ${ }^{2}$ - Christian Benedict ${ }^{1} \cdot$ Jessica Mwinyi $^{1}$. \\ Helgi B. Schiöth ${ }^{1}$
}

Published online: 30 January 2016

(C) The Author(s) 2016. This article is published with open access at Springerlink.com

\begin{abstract}
Background Currently, Roux-en Y gastric bypass (RYGB) is the most efficient therapy for severe obesity. Weight loss after surgery is, however, highly variable and genetically influenced. Genome-wide association studies have identified several single nucleotide polymorphisms (SNP) associated with body mass index (BMI) and waist-hip ratio (WHR). We aimed to identify two genetic risk scores (GRS) composed of weighted BMI and WHR-associated SNPs to estimate their impact on excess BMI loss (EBMIL) after RYGB surgery.

Methods Two hundred and thirty-eight obese patients (BMI $45.1 \pm 6.2 \mathrm{~kg} / \mathrm{m}^{2}, 74 \%$ women), who underwent RYGB, were genotyped for 35 BMI and WHR-associated SNPs and were followed up after 2 years. SNPs with high impact on post-surgical weight loss were filtered out using
\end{abstract}

Electronic supplementary material The online version of this article (doi:10.1007/s11695-016-2072-9) contains supplementary material, which is available to authorized users.

Marcus Bandstein

marcus.bandstein@neuro.uu.se

Sarah Voisin

Sarah.voisin.aeris@gmail.com

Emil K. Nilsson

Emil.nilsson@neuro.uu.se

Bernd Schultes

Bernd.Schultes@eswiss.center

Barbara Ernst

Barbara.Ernst@eswiss.center

Martin Thurnheer

Martin.Thurnheer@eswiss.center a random forest model. The filtered SNPs were combined into a GRS and analyzed in a linear regression model. Results An up to $11 \%$ lower EBMIL with higher risk score was estimated for two GRS models ( $P=0.026$ resp. $P=0.021)$ composed of seven BMI-associated SNPs (closest genes: MC4R, TMEM160, PTBP2, NUDT3, TFAP2B, ZNF608, $M A P 2 K 5$, GNPDA2, and MTCH2) and of three WHRassociated SNPs (closest genes: HOXC13, LYPLAL1, and $D N M 3-P I G C)$. Patients within the lowest GRS quartile had higher EBMIL compared to patients within the other three quartiles in both models.

Conclusions We identified two GRSs composed of BMI and WHR-associated SNPs with significant impact on weight loss after RYGB surgery using random forest analysis as a SNP selection tool. The GRS may be useful to pre-surgically evaluate the risks for patients undergoing RYGB surgery.

\author{
Christian Benedict \\ Christian.Benedict@neuro.uu.se \\ Jessica Mwinyi \\ Jessica.mwinyi@neuro.uu.se \\ Helgi B. Schiöth \\ Helgi.Schioth@neuro.uu.se
}

1 Department of Neuroscience, Functional Pharmacology, Uppsala University, Box 593, 75124 Uppsala, Sweden

2 Interdisciplinary Obesity Center, eSwiss Medical \& Surgical Center, St. Gallen, Switzerland 
Keywords Genetic risk score · Roux-en Y gastric bypass surgery $\cdot$ Obesity $\cdot$ Random forest model $\cdot$ Post-operative weight loss

\section{Introduction}

While obesity is today one of the world leading health problems, treatment options for obese patients remain limited. Roux-en Y gastric bypass (RYGB) surgery is currently one of the most efficient therapy alternatives for sustainable weight loss in obese patients [1]. However, the degree of weight loss and treatment success is highly variable and potential surgery-associated complications should not be underestimated [2-4]. Thus, the detection of reliable pre-surgical indicators for a risk-benefit assessment before the intervention would be of high value. Weight loss following RYGB surgery is influenced by genetic variants [5]. Large genome-wide association studies (GWAS) have detected several single nucleotide polymorphisms (SNP) that are associated with BMI and waisthip ratio (WHR) [6-8]. It cannot be excluded that the genetic variants factors associated with BMI may have an influence on Roux-en Y surgery induced weight loss.

The fat mass and obesity associated gene (FTO) has the strongest association to BMI as shown in a study including more than 340,000 people [7]. However, the explained variance of the FTO effect allele was earlier published to be only $0.34 \%$ [8], meaning that the remaining individual variance in BMI may be attributed to other genetic and non-genetic factors. Genetic risk scores (GRS) describe the collective impact of several potentially risk contributing SNPs by creating one continuous variable that indicates the likelihood to develop a disease or a trait, such as weight loss [9]. GRSs are calculated in a weighted or unweighted manner. While weighted GRS take the previously reported effect from of each individual SNP into account, unweighted GRS consider only the number of alleles of each SNP included in the calculation [10-12]. In the present study, we investigated the impact of BMI and WHR-associated SNPs on excess BMI loss (EBMIL) 2 years after RYGB surgery. Based on a selection of genetic variants, we developed a weighted GRS and estimated the post-surgery weight loss in dependency of the considered risk variants. To select SNPs having a relevant impact on EBMIL, the random forest statistical method was used [13]. This machine learning tool has been used previously to screen or reduce dimensions in large GWAS datasets [14-17]. In the current study, this statistical method is used for the first time to analyze and evaluate polymorphisms associated with weight loss after bariatric surgery.

\section{Material and Methods}

\section{Patients}

Two hundred and thirty-eight obese patients, characterized by a BMI $>30 \mathrm{~kg} / \mathrm{m}^{2}$, were included in the study (mean $\pm \mathrm{SD}$; age $43.2 \pm 10.8$, initial BMI $45.1 \pm 6.2 \mathrm{~kg} / \mathrm{m}^{2}, 74 \%$ women). All patients were weighted in the morning in a fasted state with light clothes but no shoes on. All participants underwent proximal or distal RYGB surgery at the Interdisciplinary Obesity Center, St. Gallen, Switzerland, and returned 2 years later to the study center for a follow-up investigation. Weight loss after surgery was determined as EBMIL, considering a BMI $>25 \mathrm{~kg} / \mathrm{m}^{2}$ as excess. The relative EBMIL was calculated as $100-[($ final BMI-25/initial BMI-25)*100] [18]. Demographic and clinical characteristics of the study population are summarized in Table 1. Percent BMI loss was calculated according to [18] and was used as a confirmatory independent variable in genetic association analyses.

The cohort and both bariatric procedures have earlier been described in detail [19]. In brief, a gastric pouch of about 20 $30 \mathrm{ml}$ was created after removing a major part of the stomach. The length to the biliopancreatic limb was set to approximately $60 \mathrm{~cm}$ in the proximal RYGB procedure and 60 to $100 \mathrm{~cm}$ in the distal procedure. Patients who obtained the operation for a second time or underwent an alternative intervention to RYGB surgery (e.g., gastric banding) were not included.

\section{Genotyping}

Genotyping was performed by the SNP\&SEQ Technology Platform at SciLifeLab, Uppsala, Sweden, using the Illumina

Table 1 Demographic and clinical characteristics

\begin{tabular}{|c|c|}
\hline \multicolumn{2}{|l|}{ Variable } \\
\hline Included patients, $n$ & 238 \\
\hline \multicolumn{2}{|l|}{ Sex, $n$ (\% cohort $)$} \\
\hline Female & $173(73)$ \\
\hline Male & $65(27)$ \\
\hline Age $^{a}$, years $(S D)$ & $43.1( \pm 10.8)$ \\
\hline $\mathrm{BMI}^{\mathrm{a}}, \mathrm{kg} / \mathrm{m}^{2}(\mathrm{SD})$ & $45.1( \pm 6.2)$ \\
\hline \multicolumn{2}{|c|}{ Surgery type, $n$ ( $\%$ followed up cohort) } \\
\hline Distal RYGB & $175(73.5)$ \\
\hline Proximal RYGB & $63(26.5)$ \\
\hline $\mathrm{BMI}^{\mathrm{b}}, \mathrm{kg} / \mathrm{m}^{2}(\mathrm{SD})$ & $28.5( \pm 3.9)$ \\
\hline $\mathrm{EBMIL}^{\mathrm{b}}, \%(\mathrm{SD})$ & $83.8( \pm 17.8)$ \\
\hline BMI loss ${ }^{\mathrm{b}}, \%$ (SD) & $36.3( \pm 8.4)$ \\
\hline \multicolumn{2}{|c|}{$\begin{array}{l}E B M I L \text { excess BMI loss, } R Y G B \text { Roux-er } \\
\text { Y gastric bypass }\end{array}$} \\
\hline \multicolumn{2}{|c|}{${ }^{\mathrm{a}}$ At baseline } \\
\hline \multicolumn{2}{|c|}{$\begin{array}{l}\text { b At 24-month post-Roux-en Y gastric by- } \\
\text { pass surgery }\end{array}$} \\
\hline
\end{tabular}


iSelect genotyping array (Illumina Inc.). All determined SNPs were in Hardy-Weinberg equilibrium. None of the variants were in linkage disequilibrium. Twenty-three BMI-associated and 12 WHR-associated SNPs were chosen for genotyping out of large GWAS, reporting the SNPs' association to BMI, respectively, WHR $[6,8]$. Further, a minor allele frequency of $>15 \%$ was required. The full list of determined genetic variants is presented in Supplementary table 1.

\section{Statistics}

The genotype of each SNP was coded based on the amount of effect alleles, i.e., 0 for no effect alleles, one for heterozygote carriers, and two for individuals carrying two effect alleles. Subsequently, SNP-associated betavalues as published by Speliotes et al. and Heid et al. were multiplied with the amount of alleles to obtain weighted SNP scores. Weighted scores were included in the random forest model [13] as predictors and EBMIL as the target variable. Ten thousand random decision trees were created. The random forest model result for the weighted BMI-associated SNPs showed, when plotted, a clear change in mean squared error (MSE) around $10 \%$ (Supplementary figure 1a). SNPs above $10 \%$ MSE were considered having a relevant influence on the model and were chosen for inclusion in the genetic risk score. The change in MSE was also seen in the random forest results for the weighted WHR SNPs, although not as clear. The cut off for $10 \%$ was therefore used in that model as well. The trajectory direction for each SNP with a MSE $>10 \%$ (i.e., if the SNP increased or decreased EBMIL) was tested by performing a preliminary linear regression analysis. The coding of a BMI or WHR-associated SNP was inverted in case an association with EBMIL increase was detected.

GRS were calculated by summing up the weighted SNP scores for all variants that induced a MSE $>10 \%$ in the random forest model according to the following formula: $\sum n$ effect alleles $\mathrm{SNPi}_{\mathrm{S}} \times$ beta value $\mathrm{SNPi}_{\mathrm{i}}(i=$ number of included SNPs in the model, $n=$ number of risk alleles). The GRSs were used as continuous variables in a multiple linear regression with EBMIL as the dependent variable, adjusting for age, sex, initial BMI, and surgery type. Student's $t$ test was used in the post hoc analysis to compare the strength of weight loss between GRS quartiles. $P$ values $<0.05$ were considered significant and, if necessary, adjusted for multiple testing according to Benjamini-Hochberg (BH) with the Q-level of $5 \%$ [20]. Analyses were performed using the CRAN package "Rattle" (Williams G. Data Mining with Rattle and R: The Art of Excavating Data for Knowledge Discovery.; 2011) in R studio (R Core Team, Vienna, Austria) and SPSS Statistics for Windows, Version 22.0 (IBM Corp. Released 2013. IBM. Armonk, NY: IBM Corp.).

\section{Results}

\section{Clinical Outcome}

Patients showed an average EBMIL of $84 \%$ (SD $18 \%$ ) 2 years after RYGB surgery. Younger patients and patients with lower initial BMI benefitted most from the intervention according to regression analyses correcting for age, BMI, sex, and surgery type $(\beta=-0.23, P<0.031$ resp. $\beta=-0.73$, $P<0.001)$. Men and women showed a similar EBMIL. The surgery type did not have a significant effect on EBMIL in this cohort. However, we observed a significant difference in the average percentage of individual BMI loss 2 years after RYGB surgery in dependency of proximal or distal surgery $(P=0.045)$. The percentage loss in BMI was stronger in individuals undergoing distal RYGB surgery $(33.1 \%)$ compared to patients who underwent the proximal surgery approach (36.7\%).

\section{Outcome of Random Forest Analyses}

Two random forest models were developed to evaluate the impact of genetic variants on EBMIL. In the first model 23 BMI-associated SNPs were included $\left(\mathrm{RFM}_{\mathrm{wBMI}}\right)$ [8]. The second model considered 12 WHR-associated SNPs $\left(\mathrm{RFM}_{\mathrm{wWHR}}\right)$ [6]. As shown in Table 2, seven variants associated with BMI and three variants associated with WHR induced a $>10 \%$ MSE and were considered in the subsequent calculation of GRS scores.

\section{Impact of GRSs on EBMIL After RYGB Surgery}

The first model, $\mathrm{RFM}_{\mathrm{wBMI}}$, consisting of BMI-associated SNPs, generated seven SNPs that were included in the BMI SNP-based GRS $\left(\mathrm{GRS}_{\mathrm{wBMI}}\right.$, closest genes: $M C 4 R$, TMEM160, PTBP2, NUDT3, TFAP2B, ZNF608, MAP2K5, GNPDA2, and MTCH2). The included patients showed GRS $_{\text {wBMI }}$ ranging from 3 to 37 , thereby carrying at least one risk allele and up to one risk allele in each of the seven genes. The GRS $_{\text {wBMI }}$ was significantly associated with EBMIL $(\beta=-0.32, P=0.026)$ in the multiple linear regression model adjusting for age, sex, initial BMI, and surgery type, indicating a $0.32 \%$ decrease of EBMIL per score unit. Maximum and minimum GRS $_{\mathrm{wBMI}}$ scores were associated with 83 and $89 \%$ EBMIL, respectively (Fig. 1a). The results were confirmed in linear regression analyses showing a significant impact of $\mathrm{GRS}_{\mathrm{wBMI}}$ on percent BMI loss $(\beta=-0.14, P=0.034)$. Investigated SNPs and associated genes are shown in Table 2 .

$\mathrm{RFM}_{\mathrm{wWHR}}$ detected three weighted WHR-associated SNPs (closest genes: HOXC13, LYPLAL1, and DNM3-PIGC) inducing a $10 \%$ MSE $>10 \%$ that were included into a GRS composed of WHR-associated SNPs $\left(\mathrm{GRS}_{\mathrm{wWHR}}\right)$. The generated $\mathrm{GRS}_{\mathrm{wWHR}}$ ranged from 0 to 19 and was associated with 
Table 2 Genetic variances included in GRS models

\begin{tabular}{cllllll}
\hline & SNP & Gene & $\begin{array}{l}\text { Minor } \\
\text { allele }\end{array}$ & $\begin{array}{l}\text { Reported } \\
\text { effect allele }\end{array}$ & $\begin{array}{l}\text { Risk allele/weight loss } \\
\text { lowering allele }\end{array}$ & $\begin{array}{l}\text { MSE } \\
(\%)\end{array}$ \\
\hline GRS $_{\text {wBMI, }}$ & rs571312 & MC4R & A & A & C & 23.16 \\
weighted BMI & rs3810291 & TMEM160 & G & A & G & 19.65 \\
& rs1555543 & PTBP2 & A & C & A & 18.58 \\
& rs206936 & NUDT3 & G & G & G & 17.58 \\
& rs987237 & TFAP2B & G & G & G & 17.49 \\
& rs4836133 & ZNF608 & A & A & A & 17.12 \\
GRS $_{\text {wwHR, }}$ & rs2241423 & MAP2K5 & A & G & G & 13.10 \\
weighted & rs1443512 & HOXC13 & A & A & A & 13.15 \\
WHR & rs4846567 & LYPLAL1 & A & G & G & 11.24 \\
& rs1011731 & DNM3- & G & G & A & 10.32 \\
\hline
\end{tabular}

The listed single nucleotide polymorphisms (SNP) induced a mean squared error (MSE) above $10 \%$ in the random forest model. These variants were subsequently included in the respective GRS model (weighted BMI model $\left(\mathrm{GRS}_{\mathrm{wBMI}}\right)$ and weighted waist-hip ratio model $\left.\left(\mathrm{GRS}_{\mathrm{wWHR}}\right)\right)$. Those alleles were defined as risk alleles that contributed least to the weight loss following Roux-en Y gastric bypass surgery. Interestingly, the A allele of the TMEM160-related SNP rs3810291 was reported to be associated with higher BMI by Speliotes et al. In our study, the allele, $\mathrm{G}$, is associated with EBMIL
EBMIL using multiple linear regression analysis $(\beta=-0.59$, $P=0.021)$. Maximum and minimum $\mathrm{GRS}_{\mathrm{wWHR}}$ scores were associated with 78 and $89 \%$ EBMIL, respectively (Fig. 1b). The findings were confirmed in regression analyses showing an association between $\mathrm{GRS}_{\mathrm{wWHR}}$ and percent BMI loss $(\beta=-0.29, P=0.028)$.

\section{Quartile Analysis of the GRSs}

To further analyze associations between the genetic composition and post-operative EBMIL, patients were divided into four quartiles based on their GRS. The score quartiles of $\mathrm{GRS}_{\mathrm{wBMI}}$ and $\mathrm{GRS}_{\mathrm{wWHR}}$ were entered as a factor in

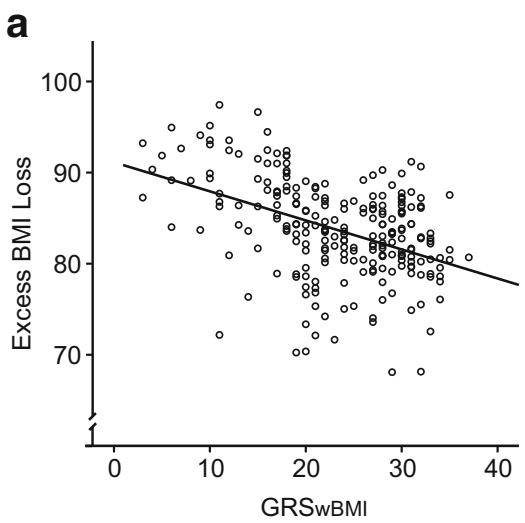

Fig. 1 The genetic risk scores (GRS), calculated on the basis of weighted single nucleotide polymorphisms associated with BMI (a GRS wBMI $_{\text {(n) }}$ and waist-hip ratio (b $\mathrm{GRS}_{\mathrm{wWHR}}$ ) estimated an up to $11 \%$ difference in excess BMI loss (EBMIL) after Roux-en Y gastric bypass surgery with increasing risk score for both models. Each GRS was inserted individually in an adjusted multiple linear regression with EBMIL as the independent variable. a $\mathrm{GRS}_{\mathrm{wBMI}}$ included genetic variants of two independent multiple linear regression analyses, correcting for age, sex, initial BMI, and surgery type. In both models, the lowest GRS quartile was associated with a significantly higher EBMIL in contrast to the other quartiles $\left(\mathrm{GRS}_{\mathrm{wBMI}}\right.$ : BH-adjusted $P<0.027, \mathrm{GRS}_{\mathrm{wWHR}}$ : BH-adjusted $P<0.048$; Fig. 2a, b). Similar results were obtained when regressing quartiles of the GRSs against percent BMI loss. In contrast to $\mathrm{GRS}_{\mathrm{wWHR}}$ quartiles, $\mathrm{GRS}_{\mathrm{wBMI}}$ quartiles showed a significant linear association with percent BMI loss $(P=0.007$ resp. $P=0.051)$. Further, patients in the first quartile lost more total BMI compared to the other quartiles $\left(\mathrm{GRS}_{\mathrm{wBMI}}\right.$ : BH-adjusted $P<0.021$, $\mathrm{GRS}_{\mathrm{wWHR}}$ : BH-adjusted $\left.P<0.032\right)$.

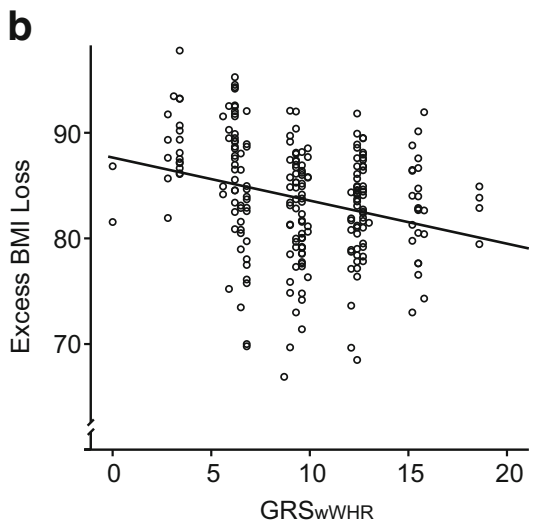

MC4R, TMEM160, PTBP2, NUDT3, TFAP2B, ZNF608, MAP2K5, GNPDA2, and MTCH2 and had a significant impact on EBMIL in a multilinear regression model adjusting for age, sex, initial BMI, and surgery type ( $P$ value of 0.026 , line equation; $y=1.1-0.32 x ; R^{2}=0.181$.

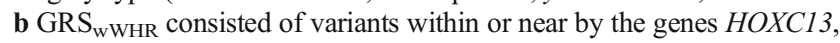
LYPLAL1, and DNM3-PIGC, $P=0.021$, line equation; $y=89.4-0.59 x$; $R^{2}=0.071$ 
Fig. 2 Quartiles of the genetic risk scores (GRS) indicate that patients with none or very few risk alleles have a higher expected excess BMI loss (EBMIL)

following RYGB surgery than patients with a higher number of risk alleles. GRSs quartiles were inserted as factors in an adjusted multiple linear regressions with EBMIL as the independent variable. a In $\mathrm{GRS}_{\mathrm{wBMI}} \mathrm{Q} 1$, the mean EBMIL was with $89 \%$ significantly different from the means of Q2, Q3, and Q4 (79 to $83 \%$ ), Benjamini Hochbergadjusted $P<0.027$. b GRS wWHR $\mathrm{Q} 1$ corresponded to a mean EBMIL of $89 \%$ compared to 81 to $82 \%$ in Q2-Q4 (Benjamini Hochberg-adjusted $P<0.048$ ). ${ }^{*} P<0.05,{ }^{*} * P<0.01$
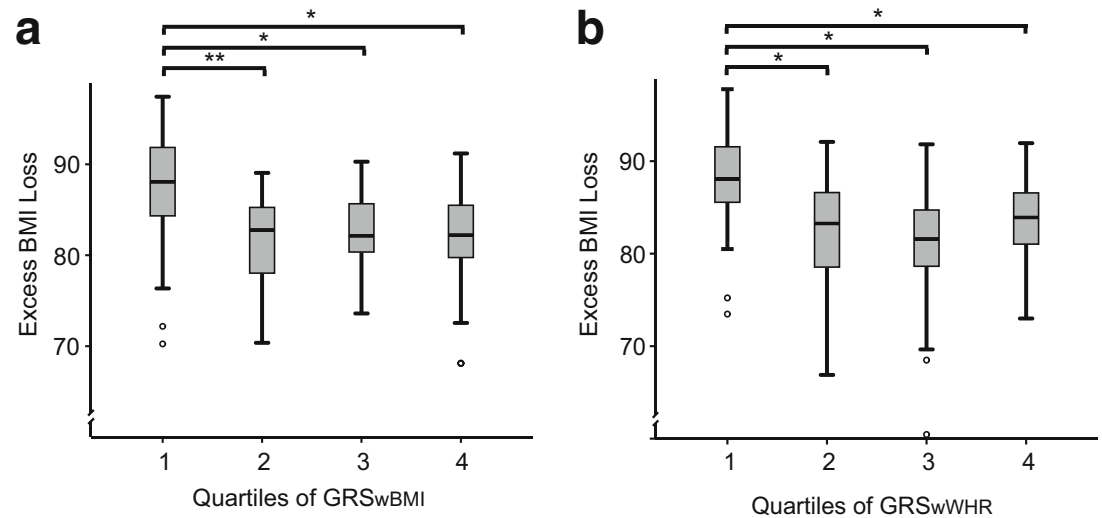

\section{Discussion}

In the present study, we demonstrate that weight loss (EBMIL) 2 years after RYGB surgery is inversely associated with two genetic risk scores composed of BMI and WHR-associated SNPs. This is the first study that developed genetic risk scores to investigate the impact of common BMI and WHR-associated SNPs on post-operative EBMIL after RYGB surgery using the random forest method as a novel selection approach for relevant SNPs. The EBMIL was up to $11 \%$ different in dependency of SNP composition in both the BMI-associated and the WHRassociated model. These findings suggest a relevant and additive effect of genetic variants on RYGB, translating into about $1.9 \mathrm{~kg} /$ $\mathrm{m}^{2}$ average weight loss. Speliotes et al. [8] reported a per-allele impact of $0.17 \mathrm{~kg} / \mathrm{m}^{2}$ for the strongest BMI-associated SNP, FTO. Our observations strengthen the hypothesis that the consideration of several risk SNPs helps to estimate post-operative weight response more accurately than isolated genetic variants and can be considered as a complement to the predictive clinical factors such as age and initial BMI.

Patients with the lowest genetic risk scores (in quartile 1; Q1) had a significantly higher EBMIL, compared to higher scoring patients in quartiles 2 to 4 (Fig. 2a, b). This observation supports the hypothesis that patients carrying none or only a small number of the risk alleles show a more efficient weight loss following RYGB surgery than carriers of multiple risk variants. Interestingly, increasing GRS do not seem to interfere proportionally with EBMIL, as the impact of GRS quartiles 2, 3, and 4 on EBMIL did not differ significantly (Fig. 2). This observation indicates that the risk alleles do not follow a strictly linear effect pattern. All tests were also performed for percent BMI loss as the independent variable and led to similar results.

Earlier studies showed that the type of surgery may have a significant impact on weight loss efficacy [21]. Therefore, we included the type of surgery as important covariate in our regression models, when evaluating the genetic risk scores that we developed. While we did not observe significant differences in EBMIL dependent on the surgery type, we did observe a significant better outcome in percentage BMI change 2 years after distal surgery. These results suggest that distal surgery may induce an efficient individual effect on weight loss, from which especially strongly obese patients may profit, despite the fact that the overall rate in EBMIL appears not to be significantly different in proximal and distal surgery.

Not all previously reported effect alleles linked to BMI and WHR in GWAS correspond to the effect allele reported in this study (Table 2). The observed differences in effect may be attributed to the difference in the investigated trait, i.e., BMI investigated in other studies compared to EBMIL investigated in our study. Furthermore, external factors, such as exercise, may impact the strength of association between SNP and trait as, e.g., observed for the gene FTO [22].

\section{Potential Mechanisms}

We report that a combination of risk alleles is linked to weight loss following RYGB surgery. When analyzing $\mathrm{GRS}_{\mathrm{wBMI}}$ and GRS $_{\mathrm{wWHR}}$-associated genes with the online tools g:Profiler [23] or String [24] for pathway analysis (and all originally included SNP-associated genes as background), no common known biological pathways were detected, suggesting that weight regulation is impacted via several not directly interfering signaling routes. All genes included in the developed GRS have previously been linked to higher BMI or WHR [6, 8]. Furthermore, we and others could show that three of these genes, namely $M C 4 R$, TFAP2B, and LYPLAL1, are associated with weight loss [25-28].

Melanocortin receptor $4(M C 4 R)$ has been implicated in eating regulation and metabolism. The protein responds to the 
agonists alpha- and beta-MSH, which is antagonized by agouti gene-related peptide in the hypothalamus regulating energy intake [29]. Studies investigating the impact of common $M C 4 R$ SNPs on bariatric surgery outcome led to contradictory results [30-33]. Activating enhancer binding protein 2 beta (TFAP2B) and lysophospholipase-like 1 (LYPLAL1) have not been linked yet to post-surgery weight loss. However, in a meal intervention study, the TFAP2B-associated SNP rs987237 was linked to weight loss in the high-fat diet group [27]. LYPLAL1 is expressed in regions known to regulate energy metabolism such as the hypothalamus and brain stem [34-36]. LYPLAL1 was associated with weight loss effects in obese patients undergoing lifestyle changes [28]. The SNP rs4846567 close to LYPLAL1 is located within an insulator segment, which may interfere with the promoter-enhancer interaction and/or block the propagation of heterochromatic structures and DNA-methylation in adjacent chromatin [37]. It can be speculated that the SNP leads to a less available promoter region and consecutively to an altered expression.

\section{Strengths and Limitations}

Previous studies that aimed to define a genetic risk score for weight loss following RYGB did not lead to significant results. These studies summed all putative risk alleles to a GRS without applying a filtering step that allows identifying genetic variants with high impact on the investigated trait $[1,38]$. Our approach using the random forest method to detect SNPs with high impact on the outcome variable appears to be an efficient and sensitive way to select relevant SNPs for the subsequently performed association analysis. It would be of value to confirm the obtained results in a larger cohort of RYGB patients.

\section{Conclusions}

Using random forest analysis as a SNP selection tool, we defined two GRSs composed of weighted BMI and WHR-associated SNPs, respectively, which are significantly associated with EBMIL after RYGB surgery. A pre-surgical determination of the GRS will help to pre-surgically evaluate to what extent patients may profit from RYGB surgery.

$B M I$, body mass index; DNM3, dynamin 3-phosphatidy linositol glycan anchor biosynthesis, class c; EBMIL, excess BMI loss; GNPDA2, glucosamine-6-phosphate deaminase 2; $G R S$, genetic risk score; $G W A S$, genome wide association study; HOXC13, homeobox c13; LYPLAL1, lysophospholipase-like 1; $M A P 2 K 5$, mitogen-activated protein kinase kinase 5; $M C 4 R$, melanocortin 4 receptor; $M S E$, mean standard error; $M T C H 2$, mitochondrial carrier 2; NUDT3, nudix-type motif 3; PTBP2, polypyrimidine tract binding protein $2 ; R F M$, random forest model; $R Y G B$, Roux-en Y gastric bypass; $S N P$, single nucleotide polymorphism; TFAP $2 B$, activating enhancer binding protein 2 beta; TMEM160, transmembrane protein 160; WHR, waist-hip ratio; $Z N F 608$, zinc finger protein 608 .

Acknowledgments We thank all of our patients who provided the blood samples and clinical information. Genotyping was performed by the SNP\&SEQ Technology Platform in Uppsala. The platform is part of Science for Life Laboratory at Uppsala University and is supported as a national infrastructure by the Swedish Research Council.

Compliance with Ethical Standards The study was performed according to the 1964 Declaration of Helsinki and its later amendments. All patients provided written informed consent to the scientific use of their clinical data sets and blood samples for genetic analyses, and the study protocol was approved by the local ethic committee of the Canton St. Gallen.

Conflict of Interest The authors declare that they have no competing interests.

Grant Information The studies were supported by the Swedish Research Foundation, the Åhlens Foundation, the Novo Nordisk Foundation, and the Swedish Brain Research Foundation.

Open Access This article is distributed under the terms of the Creative Commons Attribution 4.0 International License (http:// creativecommons.org/licenses/by/4.0/), which permits unrestricted use, distribution, and reproduction in any medium, provided you give appropriate credit to the original author(s) and the source, provide a link to the Creative Commons license, and indicate if changes were made.

\section{References}

1. Käkelä $P$, Jääskeläinen $T$, Torpström J, et al. Genetic risk score does not predict the outcome of obesity surgery. Obes Surg. 2014;24(1): 128-33.

2. Elder KA, Wolfe BM. Bariatric surgery: a review of procedures and outcomes. Gastroenterology. 2007;132(6):2253-71.

3. Griffith PS, Birch DW, Sharma AM, et al. Managing complications associated with laparoscopic Roux-en-Y gastric bypass for morbid obesity. Can J Surg. 2012;55(5):329-36.

4. de Hollanda A, Ruiz T, Jimenez A, et al. Patterns of weight loss response following gastric bypass and sleeve gastrectomy. Obes Surg. 2015;25(7):1177-83.

5. Sevilla S, Hubal MJ. Genetic modifiers of obesity and bariatric surgery outcomes. Semin Pediatr Surg. 2014;23(1):43-8.

6. Heid IM, Jackson AU, Randall JC, et al. Meta-analysis identifies 13 new loci associated with waist-hip ratio and reveals sexual dimorphism in the genetic basis of fat distribution. Nat Genet. 2010;42(11):949-60.

7. Locke AE, Kahali B, Berndt SI, et al. Genetic studies of body mass index yield new insights for obesity biology. Nature. 2015;518(7538):197-206.

8. Speliotes EK, Willer CJ, Berndt SI, et al. Association analyses of 249,796 individuals reveal 18 new loci associated with body mass index. Nat Genet. 2010;42(11):937-48.

9. Dima D, Breen G. Polygenic risk scores in imaging genetics: usefulness and applications. J Psychopharmacol. 2015;29:867-71.

10. Mega JL, Stitziel NO, Smith JG, et al. Genetic risk, coronary heart disease events, and the clinical benefit of statin therapy: an analysis 
of primary and secondary prevention trials. Lancet. 2015;385: $2264-71$.

11. Klimentidis YC, Bea JW, Lohman T, et al. High genetic-risk individuals benefit less from resistance exercise intervention. Int $\mathrm{J}$ Obes. 2015;39:1371-5.

12. Hung CF, Breen G, Czamara D, et al. A genetic risk score combining 32 SNPs is associated with body mass index and improves obesity prediction in people with major depressive disorder. BMC Med. 2015;13:86.

13. Breiman L. Random forests. Mach Learn. 2001;45(1):5-32. English.

14. Lunetta KL, Hayward LB, Segal J, et al. Screening large-scale association study data: exploiting interactions using random forests. BMC Genet. 2004;5:32.

15. Yokoyama JS, Bonham LW, Sears RL, et al. Decision tree analysis of genetic risk for clinically heterogeneous Alzheimer's disease. BMC Neurol. 2015;15(1):47.

16. Bureau A, Dupuis J, Falls K, et al. Identifying SNPs predictive of phenotype using random forests. Genet Epidemiol. 2005;28(2): 171-82. English.

17. Kautzky A, Baldinger P, Souery D, et al. The combined effect of genetic polymorphisms and clinical parameters on treatment outcome in treatment-resistant depression. Eur Neuropsychopharmacol. 2015;25(4):441-53. English.

18. Deitel M, Greenstein RJ. Recommendations for reporting weight loss. Obes Surg. 2003;13:159-60.

19. Bandstein M, Schultes B, Ernst B, et al. The role of FTO and vitamin $\mathrm{D}$ for the weight loss effect of Roux-en-Y gastric bypass surgery in obese patients. Obes Surg. 2015;25(11):2071-7.

20. Glickman ME, Rao SR, Schultz MR. False discovery rate control is a recommended alternative to Bonferroni-type adjustments in health studies. J Clin Epidemiol. 2014;67(8):850-7.

21. Brolin RE, LaMarca LB, Kenler HA, et al. Malabsorptive gastric bypass in patients with superobesity. J Gastrointest Surg. 2002;6(2): 195.

22. Loos RJ, Yeo GS. The bigger picture of FTO - the first GWASidentified obesity gene. Nat Rev Endocrinol. 2014;10(1):51-61.

23. Reimand J, Arak T, Vilo J. g:Profiler - a web server for functional interpretation of gene lists (2011 update). Nucleic Acids Research. 2011;39(Web Server issue):W307-15.

24. Jensen LJ, Kuhn M, Stark M, Chaffron S, Creevey C, Muller J, et al. STRING 8 - a global view on proteins and their functional interactions in 630 organisms. Nucleic Acids Res. 2009;37(Database issue):D412-6
25. Moore BS, Mirshahi UL, Yost EA, et al. Long-term weight-loss in gastric bypass patients carrying melanocortin 4 receptor variants. PLoS One. 2014;9(4), e93629.

26. Hatoum IJ, Stylopoulos N, Vanhoose AM, et al. Melanocortin-4 receptor signaling is required for weight loss after gastric bypass surgery. J Clin Endocrinol Metab. 2012;97(6):E1023-31.

27. Stocks T, Angquist L, Banasik K, et al. TFAP2B influences the effect of dietary fat on weight loss under energy restriction. PLoS One. 2012;7(8), e43212.

28. Delahanty LM, Pan Q, Jablonski KA, et al. Genetic predictors of weight loss and weight regain after intensive lifestyle modification, metformin treatment, or standard care in the Diabetes Prevention Program. Diabetes Care. 2012;35(2):363-6.

29. Cone RD. Anatomy and regulation of the central melanocortin system. Nat Neurosci. 2005;8(5):571-8.

30. Sarzynski MA, Jacobson P, Rankinen T, et al. Associations of markers in 11 obesity candidate genes with maximal weight loss and weight regain in the SOS bariatric surgery cases. Int J Obes. 2011;35(5):676-83.

31. Censani M, Conroy R, Deng L, et al. Weight loss after bariatric surgery in morbidly obese adolescents with MC4R mutations. Obesity. 2014;22(1):225-31.

32. Valette M, Poitou C, Le Beyec J, et al. Melanocortin-4 receptor mutations and polymorphisms do not affect weight loss after bariatric surgery. PLoS One. 2012;7(11), e48221.

33. Goergen M, Manzoni D, De Blasi V, et al. Influence of obesitysusceptibility loci (MC4R and INSIG2) on the outcome of weight loss and amelioration of co-morbidity in obese patients treated by a gastric-bypass. Bull Soc Sci Med Grand Duche Luxemb. 2011;2:724.

34. Hawrylycz MJ, Lein ES, Guillozet-Bongaarts AL, et al. An anatomically comprehensive atlas of the adult human brain transcriptome. Nature. 2012;489(7416):391-9.

35. Spiegelman BM, Flier JS. Obesity and the regulation of energy balance. Cell. 2001;104(4):531-43.

36. Ono T, Nakamura K, Nishijo H, et al. Hypothalamic neuron involvement in integration of reward, aversion, and cue signals. J Neurophysiol. 1986;56(1):63-79.

37. Ghirlando R, Giles K, Gowher H, et al. Chromatin domains, insulators, and the regulation of gene expression. Biochim Biophys Acta. 2012;1819(7):644-51.

38. Still CD, Wood GC, Chu X, et al. High allelic burden of four obesity SNPs is associated with poorer weight loss outcomes following gastric bypass surgery. Obesity. 2011;19(8):1676-83. 